Ann. Zootech., I964, 13 (2), I83-I98.

\title{
LE BESOIN EN EAU DU PORCELET : ÉTUDE DE LA CONSOMMATION D'EAU AVANT LE SEVRAGE
}

\author{
A. AUMATTRE \\ avec la collaboration technique de J. Rettagliati, M. Guichard et Huguette Dewulf \\ Station de Recherches sur l'Élevage des Porcs \\ Centre national de Recherches zootechniques, Jouy-en-Josas (Seine-et-Oise),
}

\section{SOMMAIRE}

L'étude du besoin en eau du porcelet a été entreprise sur 33 portées nées au cours de l'année 1962. Les animaux recevaient, outre le lait maternel, un aliment complémentaire sec et de l'eau de boisson à volonté jusqu'au sevrage à 8 semaines.

La quantité totale d'eau consommée (eau de boisson, eau du lait et de l'aliment) a été mesurée ; elle représente par animal durant l'allaitement environ 3 fois son poids au sevrage.

En fonction de l'âge des animaux, il existe une relation entre le besoin en eau ( $E$ en $g$ ) par unité de poids vif et le poids vif ( $\mathrm{P}$ en $\mathrm{kg}$ ) de la forme :

$$
\mathrm{E}=\frac{24^{2,8}}{\mathrm{P}}+78,7
$$

La quantité d'eau de boisson consommée est liée en général à la quantité de matière sèche consommée $(r=+0,863)$ et en particulier à la matière azotée et aux sucres du régime $\left(r=0,43^{\circ}\right.$ et $r=0,619$ à quantité de matière sèche constante), alors que la matière minérale ne semble pas jouer.

Une variation de $4^{\circ} \mathrm{C}$ de la température moyenne du local de mise bas n'a pas entraîné de modifications significatives de la consommation d'eau.

\section{INTRODUCTION}

L'eat est le premier des aliments, elle constitue 65 à 70 p. Ioo des tissus formés durant la croissance (BERGE, I953 ; MAYNARD, I954) et elle assure un rôle important dans les processus métaboliques de régulation thermique et de transfert des secrétions et excrétions. 
On s'accorde pour souligner le besoin élevé en eau des animaux après le sevrage et il a été remarqué dans presque toutes les espèces que l'ingestion d'un aliment sec entraîne une consommation d'eau : LeProvsky (I957), chez le Rat, CizEk (I96I) chez le lapin, LLoyd et al., (I962) ainsi que Morris (I962) chez le Mouton, MathieU (I962) chez le jeune veau. Une privation d'eau de $50 \mathrm{p}$. Ioo s'accompagne d'une diminution de $27 \mathrm{p}$. Ioo de la consommation d'aliment et de $50 \mathrm{p}$. Ioo de la croissance chez le Rat (Crampton et Lloyd, i954; KeAne et al., I962). Pour le Porc à l'engrais on exprime le besoin en eau par rapport à la matière sèche, et CrowthER (I93I) recommande de façon empirique de distribuer aux porcs une pâtée contenant 3 à 2,2 parties d'eau pour I de matière sèche suivant l'âge. Par contre, peu de travaux se rapportent au besoin en eau du jeune pendant 1'allaitement. Seul RUPEI. (I929) signale le besoin important en eau de boisson du veau allaité.

Les quantités d'eau consommées et les besoins en eau du porcelet sous la mère sont mal connus. On sait seulement que les quantités nécessaires sont importantes : l'eau évaporée en 24 heures pour un animal de $20 \mathrm{~kg}$ varie entre 60 et $90 \mathrm{~g}$ par $\mathrm{kg}$ vif (JACQUot et $a l$., I960) ; l'eau éliminée par l'urine est de $154 \mathrm{~g}$ par $\mathrm{kg}$ de poids vif et par 24 heures à la naissance (Mc CANCE, WIDDowson, I963); 1'eau retenue est de $600 \mathrm{~g}$ par $\mathrm{kg}$ de gain de poids entre ro et $50 \mathrm{~kg}$ (BERGE et al., I953; HENRY, I96I).

Nous avons voulu, dans un premier temps, mesurer les quantités moyennes d'eau consommées spontanément par les porcelets aux différents âges et chercher leur relation avec le poids vif et le gain de poids.

Nous avons étudié ensuite l'action possible de deux facteurs sur la consommation d'eau : 1'alimentation, en dégageant l'influence des divers nutriments et la température du local de mise bas en comparant les résultats obtenus au cours de saisons successives.

\section{MATÉRIEI, ET TECHNIQUES}

\section{A. - Animaux}

262 porcelets provenant de 33 portées de notre troupeau expérimental de race Large White, nés au cours de l'année 1962 , ont été utilisés dans l'expérience. Les animaux recevaient un aliment complémentaire ( $\left.{ }^{1}\right)$ sec granulé, dès la première semaine ainsi que de l'eau de boisson à volonté. Ils étaient sevrés à la fin de la $8^{\mathrm{e}}$ semaine et maintenus dans la loge de mise bas jusqu'au $6 \mathrm{I}^{\mathrm{e}}$ jour avec le même aliment et de l'eau ad libitum.

\section{B. - MATÉRIEL}

L'expérience a duré du 15 janvier au 30 novembre 1962 ; toutes les mises bas ayant eu lieu dans la même porcherie. La température à l'intéricur du bâtiment a été maintenue aux environs de $20^{\circ} \mathrm{C}$ par chauffage en hiver et ventilation en été. Toutefois, une certaine variation se produisant, la température moyenne de chaque semaine a été déterminée graphiquement à partir des courbes d'un thermomètre enregistreur.

Les porcelets recevaient l'eau de boisson à l'aide d'abreuvoirs siphoïdes de 50 litres réalisés à

(1) L'aliment complémentaire avait la composition suivante p. 100 : criblures de blé 30 ; orge 22 ; avoine décortiquée ro ; sucre Io ; saindoux 5 ; tourteau de soja 8 ; farine de poisson de Norvège 6 ; farine de viande 6 ; composé minéral et vitaminisé porcs 3 . Il dosait p. Ioo: matière sèche 87,0 ; matières azotées totales I8,7; matières minérales 6,3 . 
cet effet. L'auge était de dimension réduite de façon à éviter les pollutions par les animaux et limiter le gaspillage. L'eau était renouvellée trois fois par semaine; les pertes d'eau par évaporation étaient déterminées à l'aide d'un abreuvoir témoin placé dans la porcherie.

\section{C. - MESURES EFFECTUÉES}

I.es quantités d'aliment complémentaire et d'eau consommées par portée étaient mesurées chaque semaine et exprimées en moyenne par porcelet compte tenu de la variation des effectifs.

En outre, les quantités et la composition du lait étaient déterminées par les techniques précédemment décrites (SALMON-LEGAGNEUR, 1956 et $1959 a$ ).

On a pu ainsi déterminer les quantités totales de matière sèche, de matières azotées, de sucres et de matières minérales consommées. Celles-ci sont exprimées en quantités moyennes par jour et par porcelet pour chaque semaine.

Pour chaque portée une moyenne des températures journalières a été calculée pour l'ensemble de la période d'allaitement.

\section{RÉSULTATS}

\section{A. - Quantrtés D'EAU CONSOMMÉES}

Les quantités moyennes d'eau consommées sous forme d'eau de boisson, de lait et d'aliment sont rapportées au tableau $\mathrm{I}$ en fonction de l'âge et du poids des animaux.

TABLEAU I

Évolution de la quantité d'eau moyenne ingérée par porcelet au cours de l'allaitement ( $g$ par jour)

\begin{tabular}{|c|c|c|c|c|c|c|c|c|c|c|c|}
\hline Semaine & 1 & 2 & 3 & 4 & 5 & ii & 7 & 8 & $9\left(^{*}\right)$ & $\begin{array}{c}\text { Total } \\
\text { pour la } \\
\text { période } \\
(\mathrm{kg})\end{array}$ & $\begin{array}{l}\text { p. } 100 \\
\text { du total }\end{array}$ \\
\hline $\begin{array}{l}\text { Poids moyen } \\
\text { des animaux } \\
(\mathrm{kg})\end{array}$ & 2,5 & 3,7 & 4.9 & 0,3 & 8,0 & 10,4 & 13,5 & 17,2 & 20,5 & - & 一 \\
\hline Eau de boisson & 9 & 50 & 95 & 135 & 248 & $46: 3$ & 892 & 1261 & 1971 & $31, i$ & 51,6 \\
\hline Eau du lait & 472 & 516 & 521 & $5^{\prime}+7$ & 555 & 506 & $4+7$ & $37: 3$ & - - & 27,3 & 14.9 \\
\hline $\begin{array}{l}\text { Eau } \\
\text { de l'aliment }\end{array}$ & - & & $-\cdot$ & 3 & 13 & 31 & 63.3 & 95 & 92 & $\stackrel{2,1}{2}$ & 3,5 \\
\hline Eau totalc & 481 & $566 \mathrm{i}$ & 619 & 685 & 816 & $100: 3$ & 1352 & 1729 & 2063 & 60,8 & 100 \\
\hline
\end{tabular}

(*) $56^{\mathrm{e}}$ au $61^{\mathrm{e}}$ jour.

On peut remarquer que les porcelets consomment l'eau de boisson en quantité notable durant la phase d'alimentation lactée ( $\mathrm{I}^{\mathrm{re}} \mathrm{a} 4^{\mathrm{e}}$ semaine). La quantité d'eau consommée augmente sensiblement lorsque le porcelet ingère des quantités croissantes d'aliment complémentaire. 
A 6 I jours l'animal a consommé une quantité totale d'eau égale à 3 fois son poids (tabl. I).

B. - REIATION ENTRE LA QUANTITÉ D'EAU CONSOMMÉE ET LE GAIN DE POIDS

$\left.\mathrm{r}^{\circ}\right)$ Poids et gains moyens à 6r jours

Les valeurs des coefficients de corrélation entre le poids moyen ou le gain de poids d'une part et les quantités totales d'eau consommées et d'eau de boisson d'autre part, sont rapportées au tableau 2 .

\section{TABLEAU 2}

Valeurs des coefficients de corrélation entre poids ou gain de poids $\grave{a}$ 6I jours et les quantités totales d'eau ou d'eau de boisson (moyenne par porcelet)

\begin{tabular}{|c|c|c|}
\hline De 0 à 61 jours & $\begin{array}{c}\text { Eau de boisson par porcelet } \\
(k g)(31,4 \ldots 8,7)\end{array}$ & $\begin{array}{l}\text { Hau totale consomméc } \\
(\mathrm{kg})(60,8 \quad \therefore 10,4)\end{array}$ \\
\hline Poids moyen $(\mathrm{kg}) 20,5 \pm 2,8 \ldots$ & $\boldsymbol{r}=+\vdash 0,665$ & $+10,779$ \\
\hline Gain moyen $(\mathrm{kg}) 19,2=1,5 \ldots \ldots \ldots$ & $r=+0,663$ & $+0,781$ \\
\hline
\end{tabular}

Tous les coefficients sont significatifs it $\mathrm{P}<0,01$.

Elles montrent la liaison étroite existant entre la quantité d'eau consommée et le poids ou le gain de poids.

\section{0) Évolution avec l'âge}

Nous avons rapporté pour chaque semaine les quantités moyennes d'eau consommées par jour au poids vif à la fin de la semaine et observé une diminution au cours du temps (fig. I).

Nous avons ensuite essayé d'ajuster mathématiquement la courbe représentant les besoins journaliers en eau $(\mathrm{E})$ par rapport au poids $(\mathrm{P})$ en fonction de l'âge (fig. 2).

Pour cela, nous avons calculé les équations de régression linéaire et hyperbolique d'après la méthode proposée par DELAGE, LEROY et POI,Y (I953) et nous avons trouvé entre le besoin en eau $\mathrm{F}$ ( (en $\mathrm{g} /$ jour et par $\mathrm{kg}$ vif) et le poids moyen $\mathrm{P}$ en $\mathrm{kg}$ les relations:

$$
\begin{aligned}
& \mathrm{E}=\mathrm{I} 55, \mathrm{I}-4,2 \mathrm{P} \\
& \mathrm{E}=\frac{242,8}{\mathrm{P}}+78,7 .
\end{aligned}
$$




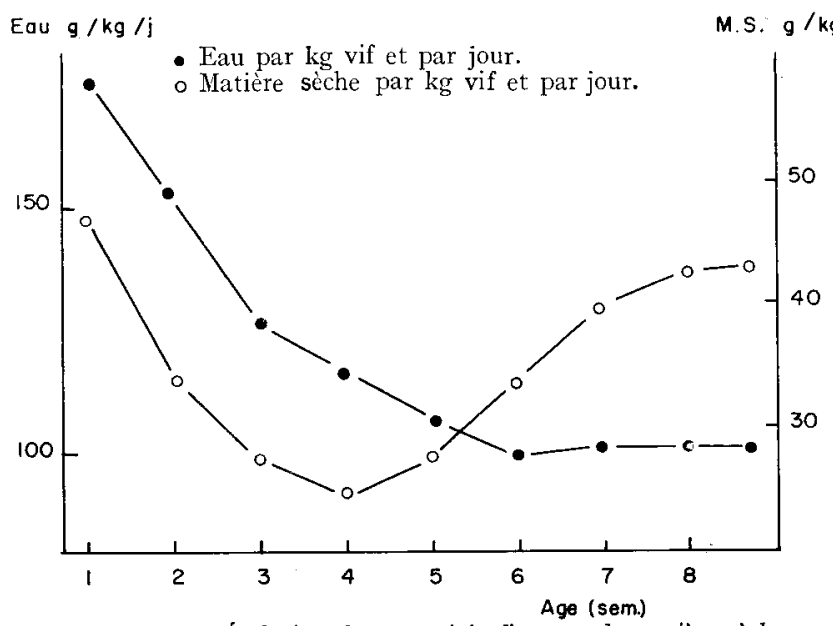

FIG. 1. - Evolution des quantités d'eau et de matière sèche consommées par jour et par $k g$ de poids vif en fonction de l'âge

\section{TABLEAU 3}

Précision des ajustements

Source de variation

Déviation par rapport à la régression hyperbolique

Déviation par rapport à la régression linéaire

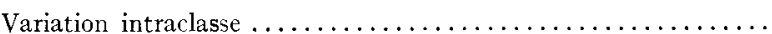

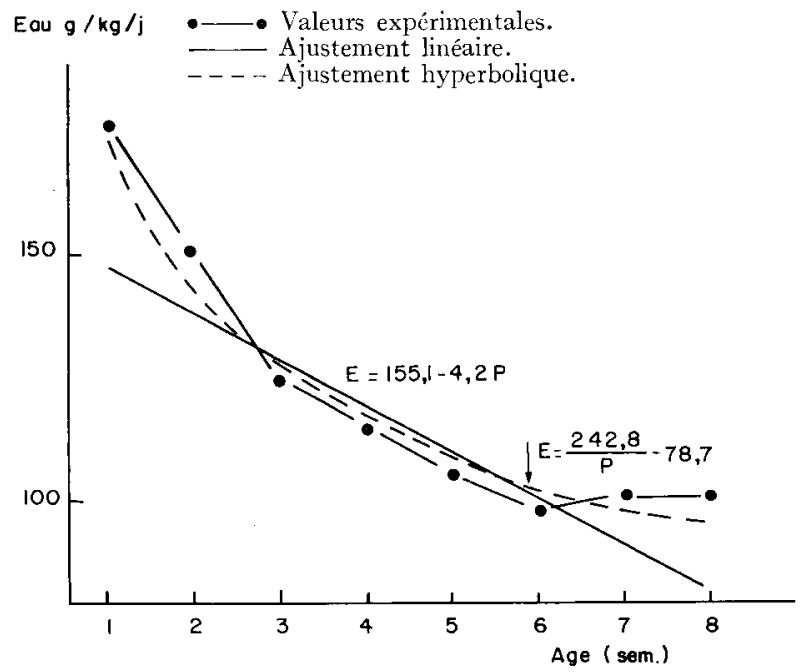

FIG. 2. - Évolution des quantités d'eau par unité de poids vif : valeurs expérimentales et ajustement 
L'analyse des variances (tabl. 3) et la figure 2 montrent que ces deux ajustements sont valables, mais que le meilleur est l'ajustement hyperbolique.

Par contre, la quantité d'eau par unité de gain de poids en fonction de l'âge semble varier suivant une loi plus complexe (fig. 3).

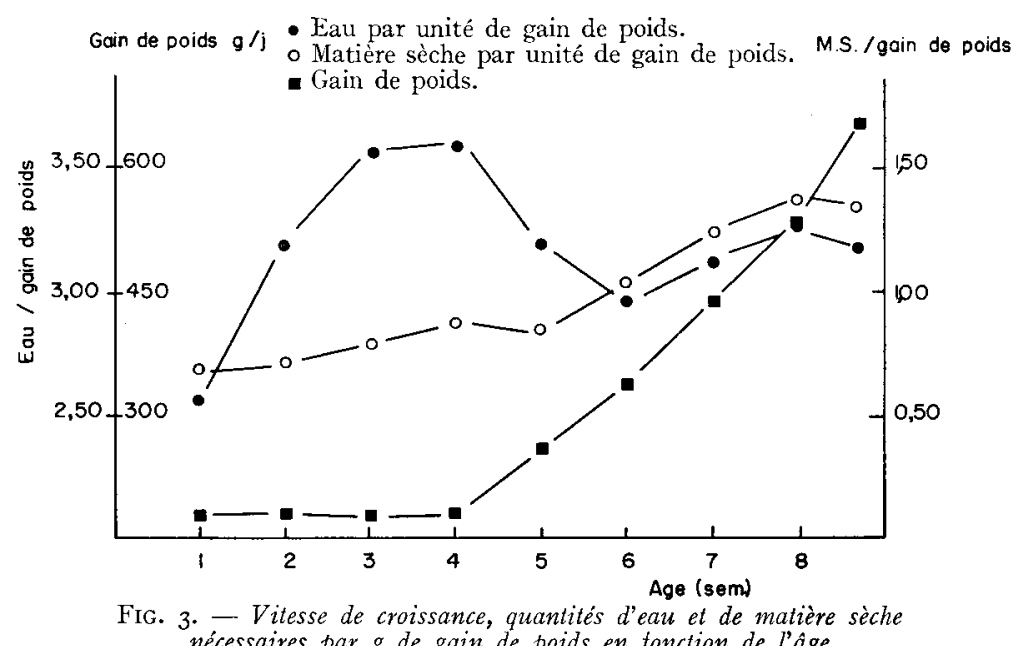

nécessaires par g de gain de poids en fonction de l'âge

\section{C. - RELATiON AVEC LA quANTité D'Aliment CONSOMMÉE}

Il était logique de chercher un rapprochement entre les quantités d'eau et d'aliment ingérées. Ces dernières peuvent agir par leur niveau général (matière sèche ou énergie), mais aussi plus spécifiquement grâce à l'action particulière d'un nutriment (azote, glucides, minéraux...). L,es différents aspects sont envisagés ici.

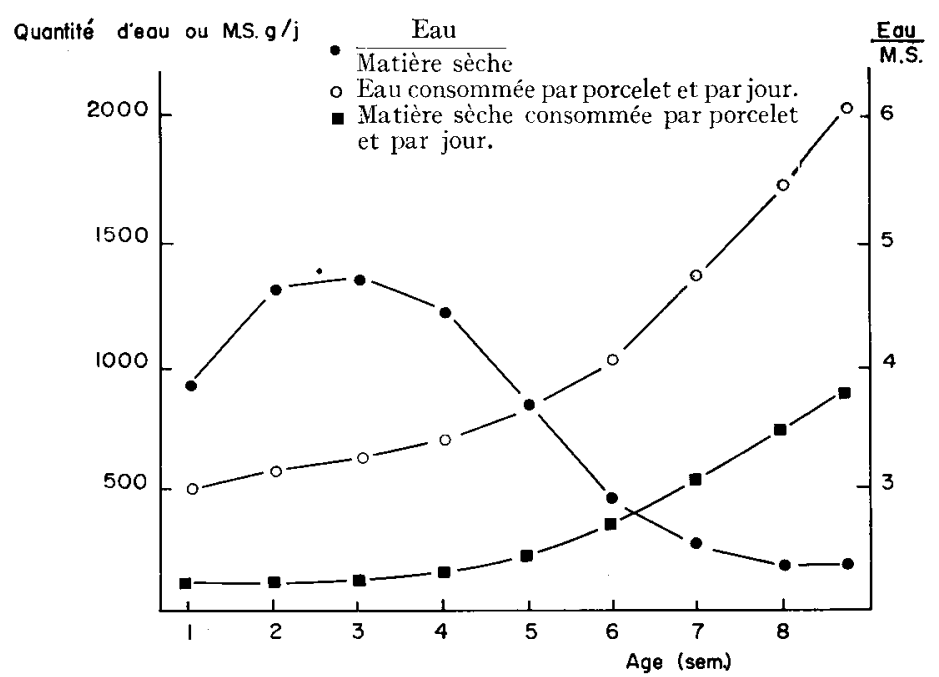

FIG. 4. - Évolution comparée des quantités totales moyennes d'eau et de matière sèche consommées ainsi que du rapport eau/matière sèche 


\section{Io) Matière sèche}

La quantité moyenne de matière sèche ingérée donne une représentation approximative de l'énergie, provenant à la fois du lait et de l'aliment, consommée par les porcelets.

La figure 4 montre comment évolue cette quantité en regard de la quantité d'eau consommée, ainsi que le rapport Eau/Matière sèche.

Les coefficients de corrélation totale entre les quantités d'eau totale et d'eau de boisson et la quantité de matière sèche ingérée sont rapportées au tableau 4. Ils sont positifs et élevés ce qui montre que les deux données varient dans le même sens, que ce soit pour différents animaux au même âge, ou pour un animal donné à différents âges.

\section{TABLEAU 4}

Corrélations entre quantité de matière sèche totale el eau totale ou eau de boisson

\begin{tabular}{|c|c|c|}
\hline Coefficient de corrélation & Inter-portées (1) & Intra-portées $\left({ }^{2}\right)$ \\
\hline Eau totale/matière sèche totale $\ldots \ldots \ldots \ldots \ldots \ldots$ & $r+=0,872$ & $r=+0,991$ \\
\hline Eau de boisson/matière sèche totale... & $r=+0,853$ & $r=+0,996$ \\
\hline
\end{tabular}

(1) Entre les valeurs moyennes par porcelet de chaque portíe.

(2) Eintre les valeurs moyennes pour les différents àges.

Tous les coefficients sont significatifs à $\mathrm{P}<0,01$.

L'étude du rapport Eau/Matière sèche présente un intérêt particulier ; la figure 4 montre que celui-ci passe par un maximum entre la $2^{\mathrm{e}}$ et $\mathrm{la} 4^{\mathrm{e}}$ semaine.

A partir de 3 semaines l'évolution indique que le porcelet a tendance à ingérer des aliments de plus en plus concentrés à mesure qu'il grandit.

Nous avons enfin rapporté la quantité de matière sèche moyenne consommée au poids vif (fig. I) et au gain de poids (fig. 3) en fonction de l'âge du porcelet.

Au cours des différentes semaines, nous observons d'une part un minimum pour la quantité de matière sèche ingérée par unité de poids $\left(4^{\mathrm{e}}\right.$ semaine) et une augmentation constante pour la quantité de matière sèche nécessaire par gramme de gain de poids.

\section{0) Matière azotée}

On trouvera au tableau 5 les quantités moyennes de matière azotée consommée par les porcelets au cours de l'allaitement.

Les coefficients de corrélation entre quantités de matière azotée et quantités d'eau sont les suivants :

Matière azotée totale/eau totale $\ldots \ldots \ldots r=+0,87 \mathrm{I} \quad \mathrm{P}<\mathrm{o,OI}$

Matière azotée totale/eau de boisson...r $=+0,760 \quad \mathrm{P}<0$, oI 


\section{TABLEAU 5}

Évolution des quantités de matière azotée totale ingérées par porcelet en fonction de l'âge ( $g$ par jour)

\begin{tabular}{c|c|c|c|c|c|c|c|c|c|c|c}
\hline \hline Sernaine & 1 & 2 & 3 & 4 & 5 & 6 & 7 & 8 & $9\left(^{*}\right)$ & $\begin{array}{c}\text { Total par } \\
\text { porcelet pour } \\
\text { la période } \\
\text { (g) }\end{array}$ & $\begin{array}{c}\text { En p. 100 } \\
\text { du total }\end{array}$ \\
\hline $\begin{array}{c}\text { Matière azotée } \\
\text { lait (1) }\end{array}$ & 36 & 35 & 37 & 38 & 40 & 37 & 34 & 30 & & 2008 & 40,1 \\
\hline $\begin{array}{c}\text { Matière azotée } \\
\text { aliment }\left(^{2}\right)\end{array}$ & - & - & 1 & 4 & 18 & 48 & 89 & 135 & 186 & 2995 & 59,9 \\
\hline $\begin{array}{c}\text { Matière azotée } \\
\text { totale }\end{array}$ & 36 & 35 & 38 & 42 & 58 & 85 & 123 & 165 & 186 & 5003 & 100 \\
\hline
\end{tabular}

(1) $\mathrm{N} \times 6,38$ pour la matière azotée du lait.

(2) $\mathrm{N} \times 6,25$ pour la matière azotée de l'aliment.

$\left(^{*}\right) 56^{\bullet}$ au $61^{e}$ jour.

Mais il faut tenir compte de ce que les quantités de matière azotée et de matière sèche ingérées sont liées. Il peut donc s'agir ici de simples variations concomitantes. Aussi avons-nous calculé également la corrélation partielle entre la matière azotée

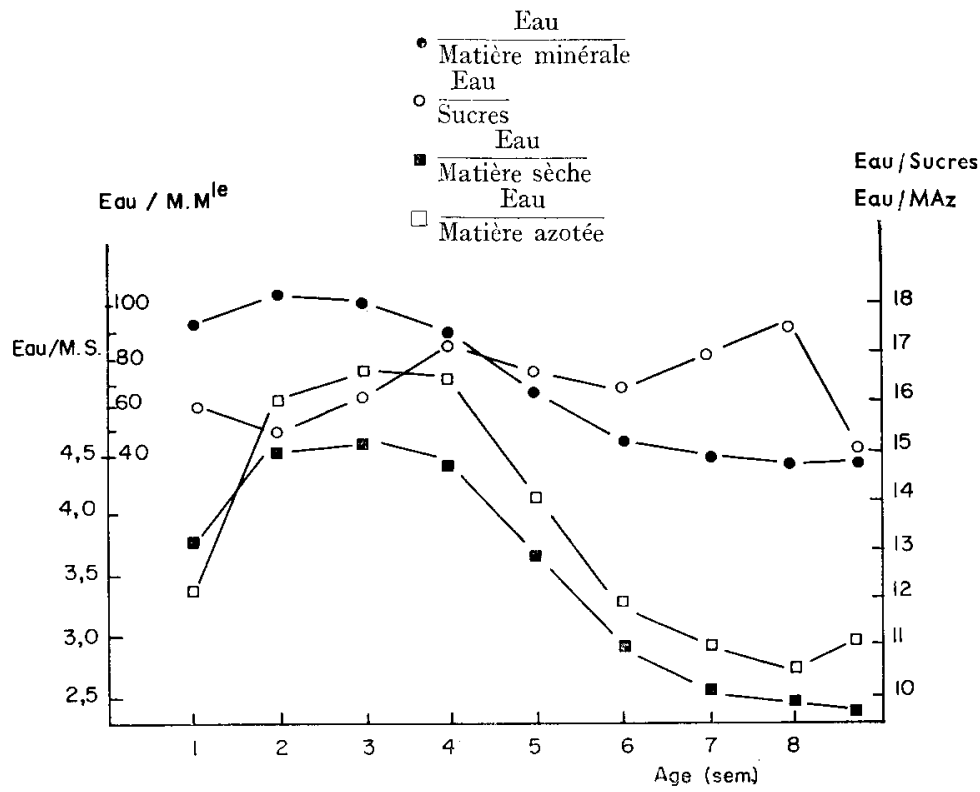

Fig. 5. - Valeur comparée en fonction du temps des rapports : $\frac{\text { eau }}{\text { matière sèche }}, \frac{e a u}{\text { matière azotée }}, \frac{\text { eau }}{\text { sucres }}, \frac{\text { eau }}{\text { matière minérale }}$. 
et l'eau de boisson, à quantité de matière sèche ingérée constante. On trouve dans ce cas $r=+0,430 \mathrm{P}<0,02$ et l'on peut penser que la quantité de matière azotée ingérée exerce un effet spécifique sur la quantité d'eai consommée.

De la même façon, compte tenu de la relation entre la matière azotée et la croissance, nous avons calculé le coefficient de corrélation partielle entre l'eau et la matière azotée, indépendamment de la vitesse de croissance.

La valeur $r=+0,6$ I $8 \mathrm{P}<0,0$ I indique qu'à vitesse de croissance constante, la quantité d'eau ingérée est d'autant plus grande que la quantité de matière azotée est élevée.

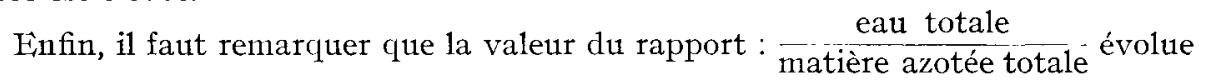
dans le même sens que le rapport $\frac{\text { eaut }}{\text { matière sèche }}$ et passe également par un maximum situé entre la $3^{\mathrm{e}}$ et la $4^{\mathrm{e}}$ semaine (fig. 5).

\section{$\left.3^{0}\right)$ Sucres}

Les quantités moyennes de sucres ingérées (lactose et saccharose) provenant du lait et de 1'aliment sont rapportées au tableau 6 .

\section{TABLIAU 6}

Erolution des quantilés moyennes de sucres (lactose + saccharose) ingérées par porcelet en fonction de liâge ( $g$ par jour)

\begin{tabular}{|c|c|c|c|c|c|c|c|c|c|c|c|}
\hline Semaine & 1 & 2 & 3 & 't & 5 & 6 & 7 & 8 & $9\left(^{*}\right)$ & $\begin{array}{l}\text { Total pour } \\
\text { la période } \\
\text { (g) }\end{array}$ & $\begin{array}{l}\text { En p. } 100 \\
\text { du total }\end{array}$ \\
\hline Lartose (lait) ...... & 30 & 37 & 38 & 38 & 39 & 36 & 31 & 25 & $\cdots$ & 1911 & 51 \\
\hline Saccharose (aliment). & - & - & 0 & 2 & 10 & 26 & 19 & 73 & 98 & 1615 & 49 \\
\hline "Sucre $"$ total $\ldots .$. & 30 & 37 & 38 & 40 & 49 & 62 & 80 & 98 & 98 & 3520 & 100 \\
\hline
\end{tabular}

$\left(^{*}\right) 56^{\mathrm{e}}$ au $61^{\mathrm{e}}$ jour.

Comme précédemment, les coefficients de corrélation totale ont été calculés entre les quantités d'eau et de sucres :

$$
\begin{array}{ll}
\text { Sucres totaux/eau de boisson } r=+0,675 & \mathrm{P}<0, \mathrm{OI} \\
\text { Sucres totaux/eau totale } \ldots . . r=+0,5 \text { Io } & \mathrm{P}<0 \text {, OI }
\end{array}
$$

Les coefficients de corrélation partielle d'autre part montrent qu'à quantité de matière sèche constante, il existe une liaison étroite entre les quantités totales de sucres et d'eau de boisson $r=+0,6 \mathrm{Ig} \quad \mathrm{P}<0,0$. L'évolution du rapport Eaau/ Sucres (fig. 5) est sensiblement différente de celle des rapports précédents. 


\section{$\left.4^{\circ}\right)$ Matière minérale}

Les quantités moyennes de matière minérale ingérées sont rapportées au tableau 7 et on note la part importante de l'aliment dans l'apport minéral total.

Les relations entre les quantités d'eau et de matière minérale sont les suivantes :

Matière minérale totale/eau de boisson $\ldots . r=+0,8$ ro $\quad \mathrm{P}<0$, or

Matière minérale totale/eau totale $\ldots \ldots . . r=+0,872 \quad \mathrm{P}<0$, or

\section{TABLEAU 7}

Évolution des quantités moyennes de matière minérale ingérées par porcelet en fonction de l'age ( $g$ par jour)

\begin{tabular}{c|c|c|c|c|c|c|c|c|c|c|c}
\hline \hline Semaine & 1 & 2 & 3 & 4 & 5 & 6 & 7 & 8 & $9\left(^{*}\right)$ & $\begin{array}{c}\text { Total pour } \\
\text { la période (g) }\end{array}$ & En p. 100 \\
\hline $\begin{array}{c}\text { Matière minérale } \\
\text { lait ........... }\end{array}$ & 5 & 5 & 6 & 6 & 7 & 6 & 6 & 5 & - & 331 & 24,5 \\
\hline $\begin{array}{c}\text { Matière minérale } \\
\text { aliment......... }\end{array}$ & - & - & 0 & 2 & 6 & 17 & 31 & 46 & 63 & 1022 & 75,5 \\
\hline $\begin{array}{c}\text { Matière minérale } \\
\text { totale............ }\end{array}$ & 5 & 5 & 6 & 8 & 13 & 23 & 37 & 51 & 63 & 1353 & 100 \\
\hline
\end{tabular}

$\left(^{*}\right) 56^{\mathrm{e}}$ au $61^{\mathrm{e}}$ jour.

Contrairement à la matière azotée et aux sucres, à quantité de matière sèche constante, 1a quantité d'eau de boisson ingérée et la quantité de matière minérale ne semblent pas liées, car on trouve dans ce cas : $r=0,130$ (N. S.).

L'évolution du rapport eau/matière minérale, représentée par la figure 5, montre en particulier un maximum puis une diminution sensiblement constante en fonction du temps.

\section{D. - INFLUENCE DE LA TEMPÉRATURE AMBIANTE}

\section{a) Températures moyennes observées}

Les valeurs ont été calculées à partir des températures moyennes par portée et sont rapportées au tableau 8. Il convient de souligner qu'en aucun cas les porcelets n'étaient exposés au soleil ni maintenus au dehors.

Nous avons distingué successivement deux périodes durant l'année :

$\left.\mathrm{I}^{\circ}\right)$ Période hiver-printemps : température intérieure moyenne inférieure à $20^{\circ} \mathrm{C}$.

$\left.2^{\circ}\right)$ Période été-attomne : température intérieure moyenne supérieure à $20^{\circ} \mathrm{C}$.

Nous observons, malgré la climatisation du local une différence d'environ $4^{\circ} \mathrm{C}$ entre les saisons. 
TABLEAUU 8

Influence de la température sur les quantités moyennes consommées par porcelet et sur le rapport eaulmatière sèche durant la période (o $a ̀$ 6r jours)

\begin{tabular}{|c|c|c|c|}
\hline Saison & $\begin{array}{l}\text { IIiver-printem]s } \\
15 \text { janvier-25 avril }\end{array}$ & $\begin{array}{l}\text { Été-automne } \\
26 \text { avril-20 novembre }\end{array}$ & $\begin{array}{l}\text { Valeur de } t \text { et } \\
\text { signification des } \\
\text { différences }\end{array}$ \\
\hline Nombre d'observations.......... & 16 & 17 & \\
\hline $\begin{array}{l}\text { Températures moyennes obser- } \\
\text { vées en }{ }^{\circ} \mathrm{C}\left({ }^{1}\right) \ldots \ldots \ldots \ldots \ldots \ldots\end{array}$ & $1703(12$ à 2.200$)$ & $21^{\circ} \quad\left(13,5\right.$ à $\left.\because 9^{\circ}\right)$ & $\begin{array}{l}t=1:=1-7 \\
\mathrm{P}<0,01\end{array}$ \\
\hline Quantité d'eau consommée $(\mathrm{kg}) \ldots$ & 60,0 & $10.3,2$ & 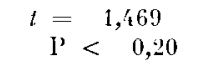 \\
\hline $\begin{array}{l}\text { Quantité de matière sèche } \\
\text { consommée }(\mathrm{kg}) \ldots \ldots \ldots \ldots \ldots \ldots\end{array}$ & 19,9 & $\because 1,9$ & $\begin{array}{l}t=1,409 \\
V^{\prime}<0,10\end{array}$ \\
\hline Eau/g de gain de poids (g) & 3,06 & 3,27 & $\begin{array}{l}t=1,755 \\
\mathrm{P}<0,10\end{array}$ \\
\hline Rapport $\frac{\text { Eau }}{\text { matière sèche }} \ldots$ & 2,922 & 2,926 & $l=0,0003$ \\
\hline
\end{tabular}

(1) Valeurs moyennes des températures journalières calculées par portée.

b) Influence de la température sur les quantités d'eau et d'aliment consommées, le rapport eau/matière sèche et la quantité d'eau par unité de gain de poids

Les valeurs observées ainsi que la signification des différences sont rapportées au tableau 8 et on peut noter en particulier l'égalité des rapports eau/matière sèche observés durant les deux périodes alors que les quantités d'eau nécessaires par unité de gain de poids (bien que non significatives) semblent plus grandes aux températures élevées.

\section{DISCUSSION}

Le besoin en eau est souvent déterminé de façon empirique, à partir du comportement et de la croissance des animaux en raison de la difficulté d'étude des bilans. La quantité totale d'eau consommée constitue donc une évaluation du besoin global en raison de sa régulation physiologique interne (LEPKOVSKY, I958). Cette quantité peut être en relation avec les phénomènes métaboliques, et en particulier la croissance, ou être déterminée par des phénomènes physiques au niveau du tube digestif. Ces différents points sont examinés. 


\section{A. - RELATIONS ENTRE LES QUANTITISS \\ D'EAU CONSOMMÉES ET IA CROISSANCE}

Nous remarquons, comme RUPEL (r929), que les animaux consomment de l'eau de boisson durant la phase lactée exclusive. Ceci semble montrer que le besoin en eau du jeune animal n'est pas entièrement couvert par le lait de la mère.

Les quantités observées sont comparables à celles signalées par différents auteurs pour le Porc après le sevrage ou pour les espèces domestiques les plus variées (tabl. 9).

\section{TABIEAU 9}

Comparaison des besoins rapportís en $g$ d'eau par $\mathrm{kg}$ oif par 24 heures

\begin{tabular}{|c|c|c|c|}
\hline Espèce & Auteur et date & Agre . Éroque ou poirls & $\mathrm{g} / \mathrm{kg}$ vif/jour \\
\hline Rat & ("RAMPTON et Llovi) (195') & 't a 8 semaines & $9 y^{\prime}$ \\
\hline Chien & KONISHI $(1960)$ & Alulte (Beanle $9 \mathrm{kgr}$ ) & 110 \\
\hline Poule & Ralen $(1961)$ & Aclulte (voncleuse) & $1 ' t$ \\
\hline Lapin & (IZLK (196;) & Arblute $(4-3 \mathrm{~kg})$ & 100 \\
\hline Veaıı & Mathinet $(196 ; 2)$ & $\begin{array}{l}\text { a semaines } \\
10 \text { semaines }\end{array}$ & $\begin{array}{r}1: 0 \\
80\end{array}$ \\
\hline Mouton & NoRRIS (I $96 \%)$ & Adulte (i ans) & 62 \\
\hline Porc & ENARD (1929) & $\begin{array}{l}11 \mathrm{~kg} \\
23 \mathrm{~kg}\end{array}$ & $\begin{array}{l}136 \\
116\end{array}$ \\
\hline Porc & Crowther (1931) & $\begin{array}{l}\text { Sevrage } \\
\text { Abattage }\end{array}$ & $\begin{array}{r}120 \\
40\end{array}$ \\
\hline l'orcelet & ATMAITRE $(19(63)$ & $\begin{array}{c}1^{\text {re }} \text { semaine } \\
\text { Sevrage } \\
\text { Ioyeme allaitement }\end{array}$ & $\begin{array}{l}177 \\
100 \\
118\end{array}$ \\
\hline
\end{tabular}

Le point le plus important et le plus caractéristique semble être la diminution suivant une loi hyperbolique de la quantité d'eau consommée par unité de poids en fonction de l'âge du porcelet. Ce phénomène entrevu empiriquement par EvvarD (I929) peut s'expliquer par le fait que le jeune animal a une croissance relative plus rapide que l'animal âgé, les tissu élaborés contiennent plus d'eau et les échanges métaboliques sont plus rapides.

Les valeurs sensiblement constantes observées de la quantité d'eau nécessaire par unité de gain de poids à partir de la $5^{\mathrm{e}}$ semaine (fig. 3) soulignent encore le fait que la quantité d'eau consommée est plus une fonction du poids que du gain de poids. 


\section{B. - RELATION AVEC LES ALIMENTS CONSOMMÉS}

La corrélation élevée et positive entre quantités d'eau et de matière sèche totale ingérées montre que les deux valeurs varient dans le même sens, mais il faut noter que 45 p. Ioo de l'eau provient du lait dont le taux d'hydratation échappe au porcelet. Il est plus intéressant de souligner la valeur du coefficient de corrélation entre les quantités d'aliment complémentaire et d'eau de boisson (tabl. 4). Une liaison analogue est signalée par de nombretux auteurs, notamment STROMINGER (I947) et Crampton et al., (1954) chez le Rat, Cizfr (I96r) chez le Lapin, Morris et al., (1962) et surtout Lioyd et al., ( 1962 ) qui ont trouvé chez le Mouton un coefficient de corrélation $r=+0,97$ entre les quantités totales d'eau et de matière sèche consommées, pour des animaux adultes.

Elle est vraisemblablement due au fait qu'une quantité d'eau bien définie est nécessaire pour assurer une bonne digestion de la matière sèche (LEPKOVSKY et $a l$., 1957). En effet, une restriction d'eau entraîne une diminution de la quantité de matière sèche ingérée ainsi que l'ont observé CraMrPTON et al., (I954), KEANE et al., (I962) et Morris et al., (Ig62); d'autre part, une quantité trop importante d'eau incorporée à l'aliment limite l'ingéré sec du Rat (KEANE, I962) ou entraîne un gaspillage chez le Porc (Crowther, I93I ; RÉrat, I963). Il semble done qu'il convient, pour assurer une consommation maximum d'aliment (AUMAîTRE et SALMON-LEGAGNEUR, Ig6I) de fournir aux jeunes animaux de l'eau de boisson à volonté, séparée de l'aliment sec, car les consommations d'aliment et d'eau n'ont pas toujours lieu simultanément (HARPER et SPIVEY, I958. Les modalités de régulation physiologique sont discutables, mais on peut penser cependant avec LEPKOvSKY et al., (I957), Cizer (Ig6r) et BAISSET et al., (I962) que l'ingestion d'aliment sec a un effet osmotique entrainant une diminution du volume des liquides extracellulaires, cette dernière provoque la sensation de soif chez l'animal et le conduit à suspendre sa consommation d'aliment.

Tout semble donc se passer comme si le porcelet, à un âge donné, réglait ses ingestions en fonction d'un rapport eau/matière sèche déterminé. Ce rapport, élevé au début de la période, passe par un maximum correspondant au moment où l'animal reçoit uniquement du lait dont la teneur en matière sèche est minimum (SALMONLEGAGNEUR, I959 a) et ingère une quantité relative de matière sèche par rapport au poids vif, minimum (fig. r). En fonction de l'âge, et à partir de la $3^{\mathrm{e}}$ semaine, le rapport diminue de façon constante, en accord avec les recommandations d'EvvaRD (I929) et de Crowther (I93I).

Lorsque l'on considère de façon séparée les principaux éléments de la matière sèche, le rôle de la matière azotée est souligné par la valeur des coefficients de corrélation totale et partielle entre quantités d'eau de boisson et de matière azotée.

Les deux quantités sont liées quels que soient l'apport de matière sèche ou la vitesse de croissance ; la matière azotée semble donc exercer une influence propre sur 1a quantité totale d'eatı consommée ainsi que le rapportent LEITCH et THOMsON (I944) qui signalent une variation du rapport eau/matière sèche chez des bœufs pour lesquels la teneur en matière azotée de la ration augmente. Des phénomènes analogues sont signalés par RUPE, chez le Veau, par Adol,PH (I933) et KEANE et al., (I962) chez le Rat ainsi que par LLOvd (1962) chez le Mouton.

Annales de Zootechnie. - I964. 
Ceci est dû à plusieurs causes :

- soit que le volume de l'urine excrétée augmente avec la quantité de protéines ingérées et donc d'urée éliminée (Lír'TCH, I944),

- soit qu'une quantité importante d'eau est nécessaire pour l'hydrolyse des protéines lors de la digestion (MAynaRD, I954),

- soit enfin que la production d'eau métabolique est très faible car peu d'éléments sont catabolisés dans la phase de croissance intense de l'allaitement.

L'influence des sucres sur la quantité d'eau consommée apparaît dans les coefficients de corrélation totale et partielle élevés entre les quantités de sucres et d'eau de boisson consommées. Flle est bien connue et réside dans la propriété des sucres à faible poids moléculaire d'entraîner une augmentation de la prise d'eau de boisson et de maintenir ainsi une pression osmotique constante (HARPER et SPIVEY, I958). Ceci est également confirmé par la valeur du rapport eau/sucres sensiblement constante quel que soit l'âge du porcelet.

Il est beaucoup plus difficile de connaitre par contre, l'effet de la matière minérale. Nos résultats diffèrent un peu de ceux d’ADOLPH (I933) et alors que le coefficient de corrélation totale entre les quantités est élevé, le coefficient de corrélation partielle à quantité de matière sèche constante n'est pas significatif. Nous ne trouvons donc pas d'effet spécifique de la matière minérale, ce qui peut paraître surprenant. Ceci peut être dû au fait que la matière minérale représente un faible pourcentage de la matière sèche ingérée, et que de plus les quantités varient peu. Toutefois, une explication apparaît dans le fait que certains minéraux (chlorure de sodium), distribués à des doses convenables, provoquent une augmentation de la rétention d'eau (CIzEK, I96I ; Mc CANCE et Widdowson, I963). L'ingestion d'eau paraissant au contraire liée à une diminution de l'eau extracellulaire, on comprend que les minéraux soient sans effet sur la consommation.

\section{C. - INFLUENCE DE LA TEMPÉRATURE}

L'écart moyen de température observé $\left(4^{\circ} \mathrm{C}\right)$ entre les deux groupes de portées hiver-printemps et été-automne a eu pour effet une légère variation des quantités d'eau et d'aliment consommées. La quantité d'eau nécessaire par unité de gain de poids est aussi légèrement plus élevée ; toutefois, les différences ne sont pas significatives.

D'autre part, le rapport eau/matière sèche est remarquablement constant. La variation de température n'a donc pas eu d'effet notable sur la quantité d'eau consommée et sur la quantité nécessaire par rapport à la matière sèche.

Ces observations sont en accord avec celles de GARNER et SANDERs (I937) qui n'ont pas trouvé de variation de la consommation d'eau de trois lots de truies allaitantes maintenues à différentes températures. Elles sont toutefois différentes de celles de EVvard (I929) qui estime que le besoin en eau des porcelets est de 30 p. roo supérieur pendant l'été ; ceci peut s'expliquer par le fait que les animaux élevés en plein air sont soumis à des amplitudes de températures supérieures à celles que nous avons observées dans un local de mise bas climatisé. 


\section{SUMMARY}

\section{WATER REQUIREMENTS OF SUCKLING PIGLETS}

A study about the voluntary water intake by suckling piglets was carried out on 262 animals born during the year 1962 and weaned at 8 weeks.

The piglets were creep fed a pelleted meal and had free access to fresh water.

The influence of different factors were studied (live weight; dry matter, protein, sugars and minerals intakes; and indoor temperature).

I) - Piglets drunk water immediatly after birth and total water consumption at weaning per unit is about threefold liveweight.

2) - The was a close relationship between water requirement ( $\mathrm{E}, \mathrm{g}$ par day per $\mathrm{kg}$ liveweight) and liveweight $(\mathrm{P}, \mathrm{kg}): \mathrm{E}=\frac{242,8}{\mathrm{P}}+78,7$ between $\mathrm{I}$ and 8 weeks.

3) - - We found a high correlation coefficient $\mathrm{P}<0,0$ I between

Total dry matter and water intakes : $r=+0,872$

Total protein and water intakes : $r=+0,87 \mathbf{I}$

Total sugar (lactose + sucrose) $: r=+0,5$ Io

Total minerals and water intakes : $r=+0,872$

Multiple correlation coefficients (dry matter constant) $(\mathrm{P}<0,0 \mathrm{I})$ between

water and protein intakes $r=+0,43^{\circ}$

water and sugar intakes $r=+0,619$

showed a close relationship between " active " nutrients and voluntary water intake.

4) - The indoor temperature variations ( $4^{\circ} \mathrm{C}$ between seasons) did not affect water intake and water requirement.

5) - Practical conclusions were drawn about the interest to give suckling piglets fresh water.

\section{RÉFÉRENCES BIBLIOGRAPHIQUES}

Adolph E. F., 1933. The metabolism and distribution of water in body and tissues. Pliysiol. Rec., 13, 336371.

Aumaitre A., Salmon-Legagneur li., ig6r. Influence de l'alimentation complémentaire sur la croissance du porcelet avant le sevrage. Ann. Zoolech., 10, i 27 -1 40.

Baisset A., Montastruc P., ig62. Recherches sur le contrôle undocrinien de la soif. Action antidipsique de l'hormone antidiurétique. Path. Biol., 10, $777-787$.

Berge S., Indrebö 'I., I953. Mjölkeproduksjon hos purker. Mell-LandbHogsk, 873, 390-424.

Cizek L. J., ig6r. Relationshi ${ }_{\mu}$ between food and water ingestion in the rabbit. Amer. J. Physiol., 201, $557-566$.

CRAmp'TON E. W., Lloyd I. E., 1954. 'The effect of water restriction of the food intake and food efficiency of growing rats. J. Nutr., 54, $22 \mathrm{I}-224$.

Crowtier C., I93I. The water requirements of swine. J. R. Agric. Soc., 92, I-5 cité par Leitcil et ThomSon (I9+4). The water economy farm animals. Nutr. Abstr. Rew., 14, 197-223.

Detage J., Leroy A. M., Poly J., i953. Une étude sur les courbes de lactation. Ann. Zootech., 2, $225-267$.

Livvird J. H., 1929. A new feeding method and standards for fattening young swine. Res. Bul. I 8 Iow, Agric. Expt. Sla., cité par Caroll W. E. et Krider J. L., 1956. Siane production, 2 éd. Mc Graw IIill. éd. New-York.

Garxer F. H., SANders II. G., 1937. The water consumption of suckling sows. J. Agric. Sci., 27,638-643

Harper A. E., SPIViy II. E., I 958. Relationship between food intake and osmotic effect of dietary carbohydrate.. Aner. J. Physiol., 193, 483-487.

ILenry M., I96r. Action du volume d'eau ingréré et du rapport sodium/potassium sur le fonctionnement surrénalien. Ann. Nutr. Parts, 15, 13 I $33^{-1} 59$.

Jaçuot R., Le Bars II., Simonvet H., I960. II. Données générales sur la nutrition et l'alimentation. I. Métabolismes et transits. J. B. Baillere, l'aris. 
KEANE K. W., et al., $19^{62}$. The addition of water to purified diets and its effect upon growth, protein efliciency ratio in the rat. $J . N u l r ., 7 \%, 18-22$.

Konishi F., Mc CAY C. M., I960. The effect of limited water on nutrient utilization. J. Nutr., 70, 528-532. LeITCH I., Thomson J. S., 1944. The water economy of farm animals. Nutr. Abstr. Rev., 14, 197-223. I.epkovsky S., Lyman R., Fifying D., 1957. Gastrointestinal regulation of water and its effects on food intake and rate of digestion. Amer. J. Physiol., 188, 327-33I.

Lloyi L. li., et al., 1962. liffect of certain forage characteristics on water intake by sheep (abstr.). J. Anim. Sci., 21, 1036.

Mathleu C. M., r962. Donnćes non publiées.

MCCANCE R. A., Winpowsox E. M., 1963. The effect of administering sodium chloride, sodium bicarbonate and potassium bicarbonate to newly born piglets. J. Physiol., 165, 569-574.

Maynard I. A., I954. Animal nutrition. $4^{\mathrm{e}}$ ed. Mc Graw IIill Book Company Inc.

Morris R. J. H., IOWARD B., Mac. FARIANE W. V., I $9^{62}$. Interaction of nutrition and air temperature with water metabolism of Merino wethers shorn in winter. Aust. J. Agr. Res., 13, 320-334.

Raucit W., i96r. Versuche zur Beurteilung des Wasserhaushaltes von Legehennen. Arch. Geflugelk., 25, $76-81$.

RÉrat A., 1963. Conférence mondiale de Zootechnie. Fédération Européenne de Zootechnie, Rome Rapp 5D, 89-105.

Rupel I. W., 1929. Raising the dairy dalf. Wisc. Agr. Expt. Sta. I3ull. no 404 cité par Cranptox et Llovd, 1954. J. Nutr., 54, $22 \mathrm{r}-224$.

Salmon-Legagneur E., i 956. La mesure de la production laitière chez la Truie. Ann. Zoolech., 5, $95^{-1}$ Io.

Salmox-Legagneur E., 1959 a. La composition du lait de Truie : premières observations sur quelques facteurs de variation. Ann. Zootech., 8, 93-112.

Strominger J. L., i 947. Yale J. Biol. Med. i9-3 cité par Cizek, i 961 . Relationship between food and water ingestion in the rabbit. Amer. J. Phy'siol., 201, 557-566. 